\title{
Reliability Modeling of Complex Machinery and Electronic Products based on the Identification of Vital Components
}

\author{
Zhaotong Wang, Yupeng $\mathrm{Li}^{*}$, Mengze Li, and Xiaoyu Zhong \\ Department of Industrial Engineering, School of Mines, China University of Mining and Technology, Xuzhou, 221116, China
}

\begin{abstract}
As the core of product architecture, the vital components of complex machinery and electronics products are of great significance in the process of product development, design, and manufacturing. However, it is very difficult to identify the vital components from a large number of components. Based on this, a vital components identification method on the basis of complex networks is proposed. First, the weighted and directed complex network (WDCN) of the relationships between the components is established. Then, the modified LeaderRank algorithm is used to identify the vital nodes in the network; this algorithm has shown low computational complexity and high calculation efficiency. Finally, a classical disease outbreak model, SIR (Susceptible-Infective-Recovered), is used to verify the correctness of the identification results. In the case of complex electric blower products, the vital components are identified using the proposed method, and the final result shows the effectiveness of this method. Such identification lays the firm foundation for the dramatic improvement of the reliability and quality of complex machinery and electronics products.
\end{abstract}

Keywords: complex networks; complex machinery and electronics products; vital components; LeaderRank algorithm; complex electric blower

(Submitted on October 20, 2018; Revised on November 15, 2018; Accepted on December 10, 2018)

(C) 2019 Totem Publisher, Inc. All rights reserved.

\section{Introduction}

The vital components of complex machinery and electronics products, as the core of the whole product architecture, play an important role in the fields of product quality, reliability, and maintenance [1]. As is well-known, the highly defective design parameters (DPs) of components will directly affect product quality, and the DPs of the vital components should be attached to the importance of product reliability [2]. The process of product failure is a dynamically changing process. The failure of vital components can accelerate this process, because the vital components can spread the failure information to other components [3]. Furthermore, the key customer requirements (CRs) are always related to the vital components, and how to transfer CRs to the function requirements (FRs) is a meaningful research topic [4]. Motivated by those descriptions, research on the vital (key) components has a significant effect on the improvement of product reliability and customer satisfaction degree.

However, it is challenging to identify those vital components from the complex machinery and electronics products that include numerous components. Many practical problems are similar to this issue. For instance, the vital spreaders in social networks should be identified to spread the information quickly [5]. Moreover, the immunization of large-degree nodes, which are usually considered more influential entities, is a highly efficient method to control the epidemic spreading [6-7]. To control the spread of diseases, identifying and controlling the key dissemination source can effectively prevent further spreading of the virus [8]. Searching for drug-target candidates in anti-infectious and anti-cancer therapies can benefit from determining the vital proteins [9]. Similarly, the quality and reliability of complex machinery and electronics products can be dramatically improved by identifying the vital components at the design stage of new products.

Recently, most research has focused on identifying the vital DPs, FRs, and other important design elements in the redesign process [4]. For example, Karsak [10] proposed an integrated fuzzy linear-programming approach to prioritize DPs.

\footnotetext{
* Corresponding author.

E-mail address: ypeng_li@163.com
} 
Similarly, Chen and Ko [11] determined the fulfillment levels of FRs and part characteristics by combining the quality function development (QFD) and failure mode and effect analysis (FMEA) into a fuzzy linear-programming model. Chang and Cheng [12] focused on identifying the influential causes of failure and assigning limited resources to serious risk items through a decision-making trial in an evaluation laboratory. Ma et al. [1] introduced an improved component-identification method, which combined QFD and FEMA to consider both the fuzziness of CRs and the failure risk of components. These studies were conducted to support product redesign rather than new product development, which is considered in this study. In the recent decade, with the continuous development of the theory of complex networks, it has constantly been applied in various aspects of product development and has achieved huge success. The main applications include product family modeling [13], product module partition [14], design change propagation [15], and searching important design elements in R\&D groups [16].

In this regard, a novel approach based on complex networks is proposed to identify the vital components of complex machinery and electronics product at the design stage. Firstly, the pre-determined components and relationships between them are mapped into a weighted and directed complex network (WDCN) model. Second, a LeaderRank algorithm [17] is modified to determine the vital components. Finally, the effects of the vital components are verified by a SIR model [18]. The vital components for a complex electric blower are identified as a case study to verify the effectiveness of the proposed method.

\section{Literature Review}

Since some related research works on identifying vital design parameters has been reviewed in Section 1, this section will focus on the method of identifying vital nodes in complex networks, especially on algorithms and their applications in engineering design.

The identification of vital individuals is very significant in many fields of society, as it allows us to better understand and utilize the structure and function of various systems [19]. In the marketing field, many issues include locating advertisements and making commercial profit for e-commercial products closely rely on the identification of vital online users [5]. To facilitate the flow of information, Chen et al. studied the issue of influence maximization wherein the social networks would be significantly affected by activating several vital individuals [20]. Some studies also have been conducted on identifying vital nodes to prevent catastrophic outages in power grids or global cascade of overload failures in the internet [21]. Furthermore, Radicchi selected the best player from the records of professional sport competitions by using complex networks [22]. Successful scientists as well as popular scientific publications can also be predicted based on coauthorship and citation networks [23-24]. Based on the above research, it can be seen that identifying vital design elements (such as the FRs, DPs, and components) to guarantee the quality and reliability of products is critical in the area of product development. The Design Structure Matrix (DSM) is a remarkable tool in the field of product development because it can solve a number of complex and difficult design problems. Browning represented a DSM-based method to solve the system decomposition and integration problem [25]. To better manage the current engineering activities, Yassine and Braha introduced a standardized DSM model to implement concurrent engineering in the development projects of complex products [26]. In order to speed up the product design efficiency, Tang et al. introduced an integrated approach based on DSM that can capture, organize, and reuse product design knowledge [27]. Fu et al. proposed a DSM-based probabilistic model that can evaluate the risk of change propagation and avoid unnecessary redesigns [28]. Nevertheless, research on DSM-based approaches to identify vital design elements is rare in existing literature.

Identifying the vital nodes in complex networks is an important research topic and can help people identify the vital elements in various systems [29]. Lv et al. summarized the state of the art on this subject [19]. Chen et al. mentioned that the simplest method to find the vital nodes in networks is to count the number of neighbor nodes; this method is called degree centrality [20]. Chen et al. proposed a new method called the LocalRank algorithm to improve the degree centrality algorithm [29]. However, the above research neglected the local interconnectedness that negatively affects the informationspreading networks. Hence, an algorithm referred to as ClusterRank is proposed to perfect the accuracy of the above methods and considers both the number of immediate neighbors and clustering coefficient of a node [20]. However, these methods are only focused on the undirected networks, while the relationship between the elements in the real world is usually directed. Brin and Page introduced a method called PageRank to evaluate the importance of web pages, and it helps effectively identify the vital nodes in directed networks [30]. Nevertheless, this algorithm requires a parameter to ensure the existence of the solutions when dangling nodes appear in the networks. To decrease the influence due to the change in parameter for the final results, Lv et al. presented an algorithm termed LeaderRank [31]. It is parameter-free and outperforms PageRank in terms of ranking effectiveness as well as robustness against manipulations and noisy data.

The network-based identification of vital nodes has also been used in engineering design. In order to predict the failures of software, Pinzger et al. compared several centrality metrics, including degree centrality, closeness centrality, and 
betweenness centrality [32]. The variant of LeaderRank was used to determine the developer's priority, and it is also effective for the prediction of reopened bugs [33]. Li et al. used the weighted LeaderRank algorithm to find the influential function modules in complex products and systems, and the SIR model was used to verify the correctness of the method [34]. Identifying the vital nodes in complex networks has not been applied to the reliability modeling of complex mechanical and electronics products.

Motivated by the above discussion, this article combined the identification of vital nodes in complex networks and reliability modeling to improve the quality and reliability of the complex mechatronic products.

\section{Complex Networks and Susceptible-Infected-Recovered Model}

\subsection{Complex Networks}

A complex network can be represented as a graph $\mathbf{G}=(\mathbf{V}, \mathbf{E})$, where the elements of $\mathbf{V}=\left(v_{1}, v_{2}, \cdots, v_{n}\right)$ are the vertices and the elements of $\mathbf{E}=\left(e_{1}, e_{2}, \cdots, e_{m}\right)$ are the links, which are used to link the vertices. A simple network model is depicted in Figure 1. Complex networks can be divided into four types, including undirected and unweighted networks, undirected and weighted networks, directed and unweighted networks, and directed and weighted networks.

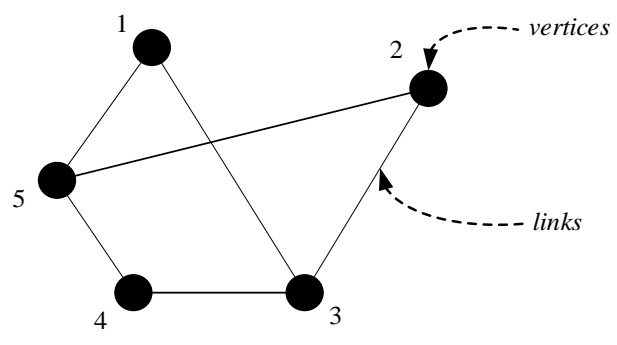

Figure 1. A simple network model

The above networks can be expressed as an adjacency matrix with $n \times n$ elements ( $n$ being the number of nodes). The elements of the adjacency matrix can be defined as follows:

$$
A_{i j}=\left\{\begin{array}{l}
w_{i j}, \text { if } i \text { and } j \text { are connceted } \\
0, \text { otherwise }
\end{array}\right.
$$

If a complex network is undirected, the adjacency matrix $\mathbf{A}$ is symmetric (i.e., $\mathbf{A}=\mathbf{A}^{\mathbf{T}}$ ). For a directed complex network, A is usually unsymmetrical.

\subsection{Random Walk Process in the Directed Complex Network}

In a directed complex network, a random-walk process is defined as the probability that a random walker on node $i$ moves toward node $j$ in the next random step. The elements of the transition matrix obtained from random-walk are described as follows:

$$
P_{i j}=\left\{\begin{array}{l}
w_{i j} / \sum_{i} w_{i j}, \text { if } i \text { and } j \text { are connected } \\
0, \text { otherwise }
\end{array}\right.
$$

Where $w_{i j}$ is the element of the adjacency matrix in the directed network. Generally, the random-walk process can be modeled using a Markov chain. After randomly walking for a sufficiently long time, the probability that the random walker is at a specific node $i$ (represented as $\pi_{i}$ ) does not change over time.

\subsection{Susceptible-Infected-Recovered Model}

The SIR model is one of the most classical models in the research field of epidemics, in which "S" represents "susceptible", "I" represents "infected", and "R" represents "recovered". In the SIR model, the population are divided into three categories. 
The "susceptible (S)" are the healthy people who are susceptible to the disease. The "infected (I)" are the people infected with an infectious disease who can spread the disease to the susceptible. The "recovered (R)" are the people who have recovered from the infectious disease and will not be infected again. In the social network, if an infected person has frequent social behaviors, then this person can accelerate the spread of the disease; however, if one person has few social behaviors, then this person may decelerate the diffusion of the disease.

Thus, in the mathematical model, an individual will be infected to measure the influence of this individual, because an individual who has huge influence can cause many people to get infected. Based on the above viewpoints, in the field of information communication and social networking, the SIR model is often the standard tool used to measure the importance or key degree of an individual [31]. Yang et al. [18] considered the reality of the spread of virus and made some improvements on the SIR model, making the model better and scientific in measuring importance degree of nodes. This study will also use this model to measure the importance degree of vital components of complex machinery and electronics products. A simplified network-based SIR model is as shown in Figure 2. At one moment, the infected can infect the susceptible with a certain probability " $\beta$ " (such as nodes 1 to 3 ), and the infected can also become the recovered with a certain probability " $\gamma$ " (such as nodes 1 to 4 ). The spread process of the disease will end when there are none infected in the network (all the infected have become the recovered).

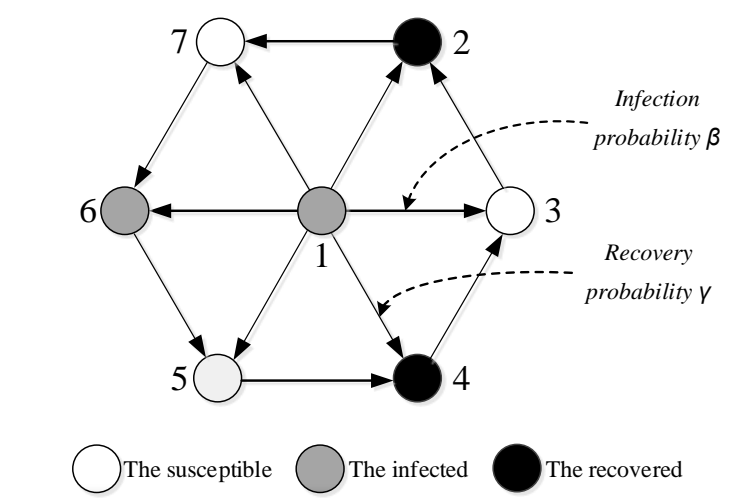

Figure 2. Schematic diagram of SIR model infection based on network

\section{Modeling}

A methodology framework has been proposed to solve the identification of the vital components within complex machinery and electronics products, as shown in Figure 3. This framework includes three steps:

Step 1 Establish the relation network of the components within complex machinery and electronics products according to the relationships between components.

Step 2 Identify the vital nodes in complex networks using the LeaderRank algorithm, where the vital nodes represent the vital components.

Step 3 Verify the efficiency of the method proposed in this study by using the network-based SIR model.

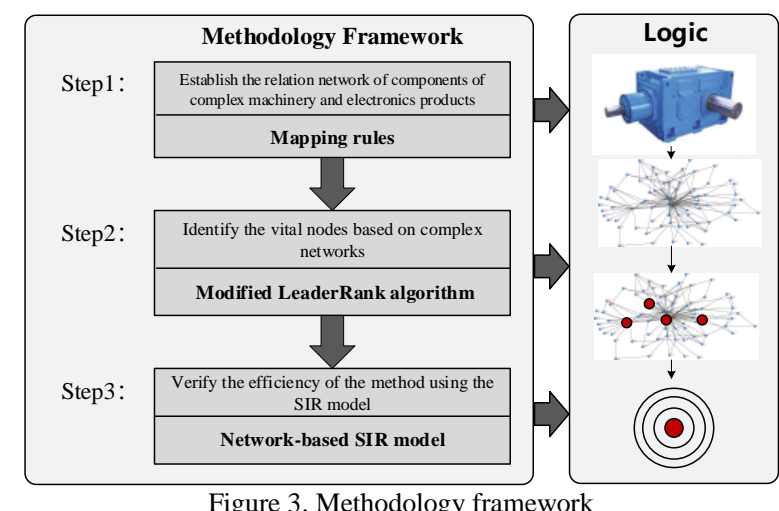

Figure 3. Methodology framework 


\subsection{Establish the Relation Network of Components of Complex Machinery and Electronics Products}

Modeling a scientific and reasonable relation network for components of complex machinery and electronics products lays a key foundation for the identification of vital components. Based on this, three mapping rules are given as follows: (1) the components of products are mapped into the nodes in complex networks; (2) the relationships between components are mapped into the edges in complex networks; (3) the comprehensive weights of the relationships are mapped into the weights of edges in networks.

The physical relationship between components of complex machinery and electronics products mainly includes connection types and geometric position. Accordingly, two aforementioned physical relationships are emphatically considered in the process of modeling the relation network of components. The types of these two physical relationships and their weights in network modeling, as shown in Table 1 and Table 2, are determined by the expert scoring method. In order to obtain the comprehensive importance of the relation between components, the various physical relations between the components need to be merged to determine the importance of the correlation. The calculation formula of the comprehensive importance of the relationship between components is as follows:

$$
E_{i j}=\sum_{k=1}^{2} w_{k i j} \cdot w_{k}
$$

Where $E_{i j}$ represents the importance degree of the relationship between component $i$ and component $j$, $w_{k i j}$ represents the weight of the $k^{\text {th }}(k=1,2)$ physical relationship between components $i$ and component $j$, and $w_{k}$ represents relative weight of the $k^{\text {th }}(k=1,2)$ physical relationship. The weights $w_{1}$ and $w_{2}$ are $2 / 3$ and $1 / 3$ respectively through the expert scoring method.

Table 1. Connection type and weight between components

\begin{tabular}{|c|c||c|c|}
\hline Connection type & Weight & Connection type & Weight \\
\hline Electronic linkage & 9 & Bearing connection & 9 \\
\hline Pin connection & 5 & Screw fastening & 5 \\
\hline Threaded connection & 5 & Buckles connection & 3 \\
\hline Slip connection & 1 & Gearing connection & 1 \\
\hline Shaft lock connection & 1 & No connection & 0 \\
\hline
\end{tabular}

Table 2. Geometric position and weight between components

\begin{tabular}{|c|c||c|c|}
\hline Geometric position & Weight & Geometric position & Weight \\
\hline Contain & 9 & Surface contact & 5 \\
\hline Line contact & 3 & Point contact & 1 \\
\hline No contact & 0 & & \\
\hline
\end{tabular}

\subsection{Identify the Vital Nodes based on the Complex Networks}

The PageRank is a classical algorithm utilized to identify vital nodes in directed networks. However, the ranking effectiveness and robustness against manipulations and noisy data of this algorithm change with the change in the parameters [17]. To solve this issue, Lv et al. [31] devised an adaptive and parameter-free algorithm termed LeaderRank. They proved that LeaderRank outperforms PageRank in terms of ranking effectiveness, as well as robustness against manipulations and noisy data. Compared to PageRank, a ground node connecting to every node through bidirectional links (see Figure 4 for illustration) is added into the network with $N$ nodes and $M$ directed edges in the LeaderRank algorithm. Thus, the network becomes a strongly connected network comprising $(N+1)$ nodes and $(M+2 N)$ directed edges. At this time, the transition matrix obtained through the random-walk process does not need to be corrected unlike PageRank, which adds a parameter. Because dangling nodes do not exist in the network, LeaderRank is termed as a parameter-free algorithm.

Moreover, LeaderRank is an adaptive algorithm. In the PageRank algorithm, the probability that a user does not follow the hyperlink and rather opens a webpage randomly in the webpage network is a constant, which is usually equal to 0.15 . However, the probability that the web pages with minority hyperlinks leads to the opening of a new webpage randomly through a ground node is higher than the web pages with majority hyperlinks in the LeaderRank algorithm. LeaderRank is closer to the actual situation, which can adjust the probability of visiting new web pages automatically based on the 
difference in the degrees of the nodes. This mechanism is referred to as the adaptivity of LeaderRank in this study. This adaptivity can be used to effectively improve the stability of the ranking and robustness of the algorithm [31].

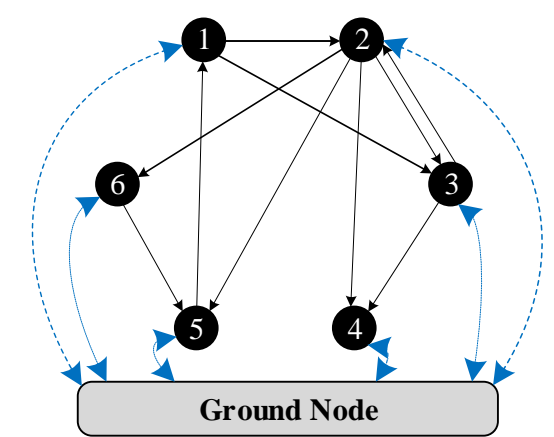

Figure 4. Illustration of the addition of ground node

Considering Figure 4 as an example, the flow diagram of the LeaderRank algorithm is demonstrated in Figure 5, and the specific steps are as follows:

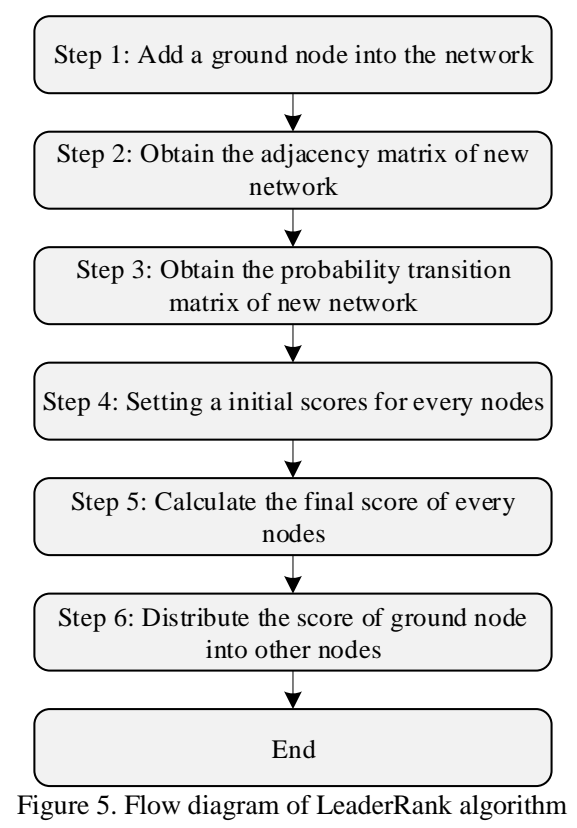

Step 1 A ground node is added into the network illustrated in Figure 4.

Step 2 The adjacent matrix $\mathbf{A}$ of the new network is obtained by Equation (1).

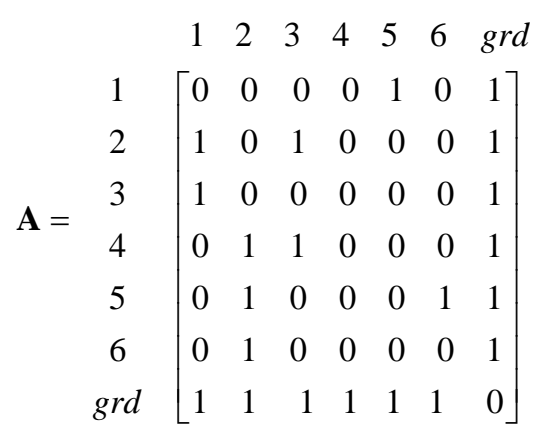

Where node grd represents the ground node. 
Step 3 The transition matrix $\mathbf{P}$ of the new network is obtained by Equation (2).

Step 4 Assume that all the initial scores of nodes are equal to $1 / n$ except the ground node, where $n$ is the number of nodes in the initial network. The initial score of the ground node is equal to 0. Assume $\boldsymbol{\pi}^{\mathbf{T}}$ is the stationary row vector of $\mathbf{P}$ and the initial value of $\boldsymbol{\pi}^{\mathbf{T}}$ is equal to the initial score of nodes. Then, proceed to the next step.

$$
\mathbf{P}=\begin{gathered}
1 \\
1 \\
2 \\
3 \\
4 \\
5 \\
6 \\
6 \\
\operatorname{grd}
\end{gathered}\left[\begin{array}{ccccccc}
0 & 0 & 0 & 0 & 1 / 2 & 0 & 1 / 6 \\
1 / 3 & 0 & 1 / 3 & 0 & 0 & 0 & 1 / 6 \\
1 / 3 & 0 & 0 & 0 & 0 & 0 & 1 / 6 \\
0 & 1 / 4 & 1 / 3 & 0 & 0 & 0 & 1 / 6 \\
0 & 1 / 4 & 0 & 0 & 0 & 1 / 2 & 1 / 6 \\
0 & 1 / 4 & 0 & 0 & 0 & 0 & 1 / 6 \\
1 / 4 & 1 / 3 & 1 & 1 / 2 & 1 / 2 & 0
\end{array}\right]
$$

Step 5 The relationship of $\boldsymbol{\pi}^{\mathbf{T}}$ and $\mathbf{P}$ can be expressed as Equation (6), and the elements of $\boldsymbol{\pi}^{\mathbf{T}}$ will be stationary after a longstanding iteration as the matrix $\mathbf{P}$ is a Markov transition matrix. The final score of nodes is equal to the final value of elements in

$$
\boldsymbol{\pi}^{\mathrm{T}}=\boldsymbol{\pi}^{\mathrm{T}} \mathbf{P}
$$

Step 6 The score of the ground node is averagely assigned to the other nodes when $\pi^{\mathrm{T}}$ achieves convergence. The final score of each node is termed the leadership score $L$, which is defined as follows:

$$
L_{i}=\pi_{i}+\frac{\pi_{g r d}}{N}
$$

Where $\pi_{i}$ and $\pi_{g r d}$ are the elements of $\pi^{\mathrm{T}}$ at the steady state. Clearly, the nodes with a higher LeaderRank score will be more vital in the network [31]. For the network shown in Figure 4, the LeaderRank scores of all nodes are obtained: $\mathbf{L}=$ $(1.043,1.083,0.894,1.033,1.103,0.844)$.

\subsection{Verify the Efficiency of the Method using SIR Model}

The components often fail due to wear, fracture, and corrosion. The failure has a certain probability spread to other components and causes them to fail. By analogy, a component failure may cause a cascading failure. In addition, a certain failure component also has a certain probability to become a scrapped component, and then the scrapped component does not deliver the failure or accept the failure transmission. This process is similar to the spread of disease, and the failure information is similar to the disease. Based on the above analysis, this paper uses the SIR model to verify the efficiency of the identification of vital components. The main steps are as follows:

(1) Select the network nodes represented by key parts or non-critical parts for disease infection.

(2) Measure the comprehensive influence of a network node by running a network-based SIR model, which is represented by the total number of network nodes infected by the selected node.

(3) If the vital nodes obtained by the algorithm are consistent with the results detected by the network-based SIR model, the validity and correctness of the method can be explained.

\section{Case Study}

In order to verify the effectiveness of the proposed method in this study, the complex electric blower (see Figure 6) is selected as a case study to identify the vital components in this product. 


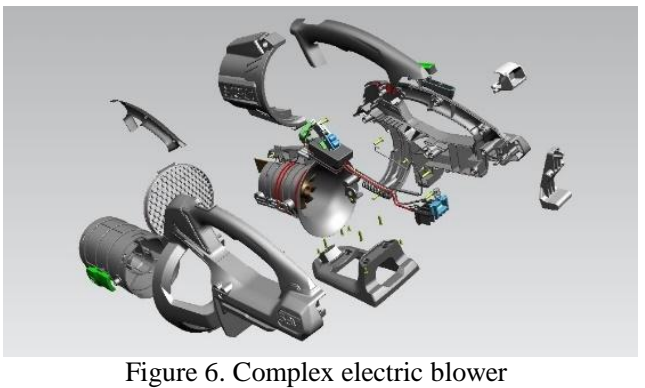

\subsection{Establish the Relation Network of the Components of Complex Electric Blower}

According to the mapping rules proposed in Section 3.1, a complex network model of electric blower components can be established, as shown in Figure 7. The components represented by nodes are presented in Table 3.

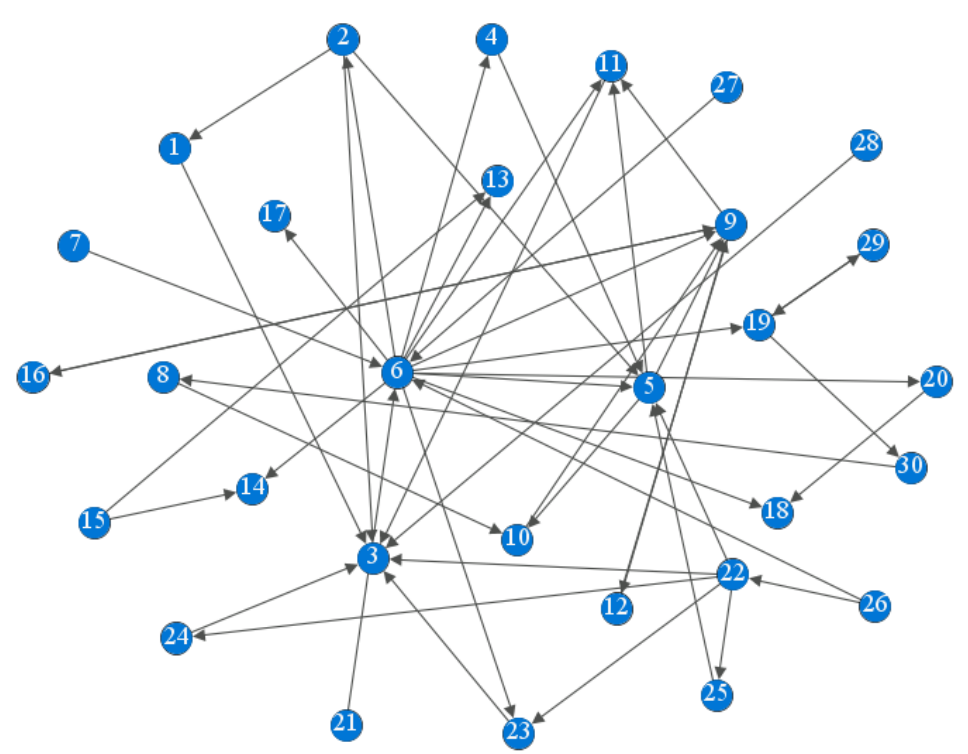

Figure 7. WDCNs of the components of complex electric blower

Table 3. Components of complex electric blower

\begin{tabular}{|c|c||c|c|}
\hline Node & Component & Node & Component \\
\hline 1 & Rubber ring 1 & 16 & Zoom button \\
\hline 2 & Left casing protector & 17 & Lever \\
\hline 3 & Movement & 18 & The trigger \\
\hline 4 & Wire rack & 19 & Power connector \\
\hline 5 & Channel components & 20 & Trigger spring \\
\hline 6 & Left casing & 21 & Right hand \\
\hline 7 & Left hand & 22 & Right casing \\
\hline 8 & Capacitance & 23 & Ventilation network \\
\hline 9 & PCBS & 24 & Rubber ring 2 \\
\hline 10 & Conductor & 25 & Right chassis cover \\
\hline 11 & Heat-shrinkable tube R1 & 26 & The base \\
\hline 12 & Speed dial button & 27 & Hooks \\
\hline 13 & Release button & 28 & Ram \\
\hline 14 & Battery locking buckle & 29 & Battery pack \\
\hline 15 & Battery pack latch spring & 30 & Heat contraction tube R2 \\
\hline
\end{tabular}




\subsection{Identify the Vital Nodes in Network based on the LeaderRank Algorithm}

The LeaderRank algorithm introduced in Section 3.2 is implemented with a console application of Visual Studio $2017 \AA$ on a computer with the following specifications: Intel (R) Core TM i-5-5200U CPU at 2.20 GHz, 4.0 GB of RAM, and Windows 8.1. In Figure 8, the final LeaderRank (LR) scores of the nodes are obtained. Table 4 lists the scores. From Figure 8 and Table 4, the vital nodes in the complex network of components are obtained, that is, 3, 9, 11, 6, 5, and 10, respectively corresponding to the movement, PCBS, heat-shrinkable tube R1, left casing, channel components, and conductor as the vital components.

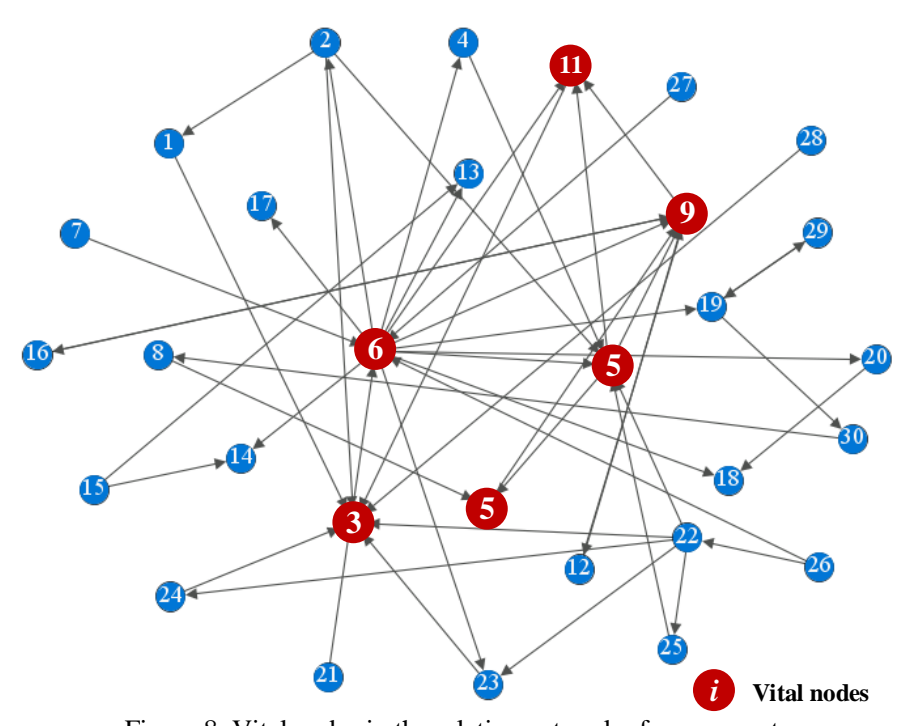

Figure 8. Vital nodes in the relation network of components

Table 4. LeaderRank score of nodes in network

\begin{tabular}{|c|c||c|c|}
\hline Node & LeaderRank Score & Node & LeaderRank Score \\
\hline 3 & $\mathbf{2 . 4 1 1 4 2 5}$ (Top 20\%) & 23 & 0.829938 \\
\hline 9 & $\mathbf{2 . 2 5 3 5 4 6}($ Top 20\%) & 22 & 0.798697 \\
\hline 11 & $\mathbf{1 . 4 6 5 6 2 8}$ (Top 20\%) & 1 & 0.787491 \\
\hline 6 & $\mathbf{1 . 3 1 2 1 4 5}($ Top 20\%) & 24 & 0.760664 \\
\hline 5 & $\mathbf{1 . 2 7 8 0 7 4}($ Top 20\%) & 25 & 0.760664 \\
\hline 10 & $\mathbf{1 . 2 3 3 5 3 7}($ Top 20\%) & 2 & 0.753872 \\
\hline 12 & 1.162409 & 4 & 0.753872 \\
\hline 16 & 1.162409 & 15 & 0.753872 \\
\hline 19 & 1.041566 & 17 & 0.753872 \\
\hline 8 & 0.972291 & 20 & 0.753872 \\
\hline 18 & 0.959659 & 7 & 0.684597 \\
\hline 29 & 0.917686 & 21 & 0.684597 \\
\hline 30 & 0.917686 & 26 & 0.684597 \\
\hline 13 & 0.891063 & 27 & 0.684597 \\
\hline 14 & 0.891063 & & 0.684597 \\
\hline
\end{tabular}

\subsection{Verify the Efficiency of the Proposed Method based on SIR Model}

In order to evaluate the effectiveness of the LeaderRank algorithm, the solution results of the algorithm are tested by the SIR model. The vital nodes (top 20\%) of 3,11, and 10 and non-vital nodes of 29 and 21 are selected to compare the influence of those nodes in the whole product and then prove the validity of the method. By running the program, the results of the comparative analysis are obtained, as shown in Figure 9. 
It can be seen that the result of the identification of vital components using the SIR model is the same as the result using the method proposed. Namely, the larger the LeaderRank value of the network node, the greater the influence of the node. Through the validation of the SIR model, the effectiveness and correctness of the proposed method in identifying vital components of complex machinery and electronics products are illustrated.

Furthermore, from the perspective of engineering reliability, the vital components of the movement, PCBS, heatshrinkable tube R1, left casing, channel components, and conductor are the easiest wear components in the complex electric blower products. If those vital components turn to failure, that failure information can lead to a widespread failure in the entire product. Therefore, these components can be regarded as the vital components of the complex electric blower. During the product design or manufacturing process, these vital components should be focused on to avoid uncontrollable design changes. The identification of vital components can improve product reliability and extend the service life of products.

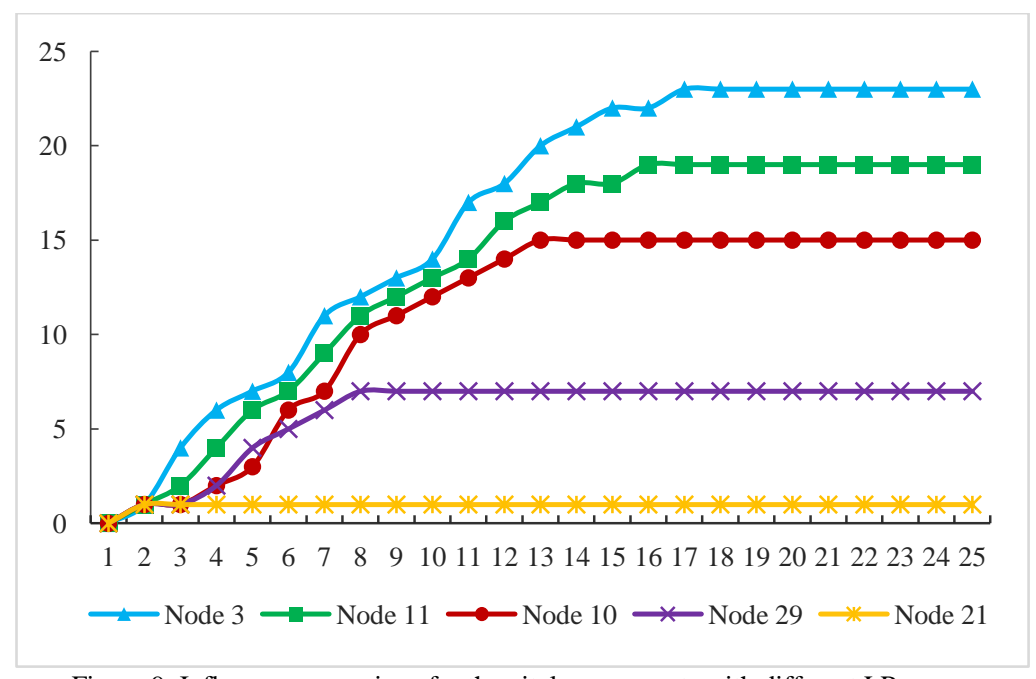

Figure 9. Influence comparison for the vital components with different LR scores

\section{Conclusions}

A method based on complex networks for the identification of vital components in complex machinery and electronics products is proposed in this study. This method considers both the connection types of components and the geometry position of two factors. Firstly, the WDCNs of the relationships between components is established, and then the LeaderRank algorithm is used to identify vital nodes of complex network. Finally, the proposed method is applied for identifying the vital components of the complex electric blower. The result indicates that the proposed method is effective and reliable. Furthermore, the proposed method also has advantages of low computational complexity and high computational efficiency. The final results can provide important basis for product design changes, product reliability improvement, and manufacturing and assembly of vital components. However, there are also some deficiencies in this study that will be the focus of future research, including the identification of key parts in non-mechanical products.

\section{Acknowledgements}

This project was supported by the Fundamental Research Funds for the Central Universities (No. 2015QNA41).

\section{References}

1. H. Ma, X. Chu, D. Xue, and D. Chen, "Identification of to-be-improved Components for Redesign of Complex Products and Systems based on Fuzzy QFD and FMEA," Journal of Intelligent Manufacturing, pp. 1-17, 2016

2. J. H. Shin, D. Kiritsis, and P. Xirouchakis, "Design Modification Supporting Method based on Product Usage Data in Closedloop PLM," International Journal of Computer Integrated Manufacturing, Vol. 28, No. 6, pp. 551-568, 2015

3. I. Alloui, S. Cîmpan, and H. Verjus, "Towards Software Architecture Physiology: Identifying Vital Components," in Proceedings of Software Architecture, WICSA 2008, Seventh Working IEEE/IFIP Conference on IEEE, pp. 293-296, 2008

4. H. B. Yan and T. Ma, "A Group Decision-Making Approach to Uncertain Quality Function Deployment based on Fuzzy Preference Relation and Fuzzy Majority," European Journal of Operational Research, Vol. 241, No. 3, pp. 815-829, 2015

5. L. Akritidis, D. Katsaros, and P. Bozanis, "Identifying the Productive and Influential Bloggers in a Community," IEEE Transactions on Systems Man \& Cybernetics Part C, Vol. 41, No. 5, pp. 759-764, 2011

6. W. J. Bai, T. Zhou, and B. H. Wang, "Immunization of Susceptible-Infected Model on Scale-Free Networks," Physica A 
Statistical Mechanics \& Its Applications, Vol. 384, No. 2, pp. 656-662, 2007

7. R. Pastorsatorras and A. Vespignani, "Immunization of Complex Networks," Physical Review E Statistical Nonlinear \& Soft Matter Physics, Vol. 65, No. 3, pp. 106-126, 2002

8. J. Liu, Q. Xiong, W. Shi, W. Shi, and K. Wang, "Evaluating the Importance of Nodes in Complex Networks," Physica A Statistical Mechanics \& Its Applications, Vol. 452, pp. 209-219, 2016

9. P. Csermely, T. Korcsmáros, H. J. Kiss, G. London, and R. Nussinov, "Structure and Dynamics of Molecular Networks: A Novel Paradigm of Drug Discovery: A Comprehensive Review," Pharmacology \& Therapeutics, Vol. 138, No. 3, pp. 333-408, 2013

10. E. E. Karsak, "Fuzzy Multiple Objective Programming Framework to Prioritize Design Requirements in Quality Function Deployment," Computers \& Industrial Engineering, Vol. 47, No. 2-3, pp. 149-163, 2004

11. L. H. Chen and W. C. Ko, "Fuzzy Linear Programming Models for New Product Design Using QFD with FMEA," Applied Mathematical Modelling, Vol. 33, No. 2, pp. 633-647, 2009

12. K. H. Chang and C. H. Cheng, "Evaluating the Risk of Failure using the Fuzzy OWA and DEMATEL Method," Journal of Intelligent Manufacturing, Vol. 22, No. 2, pp. 113-129, 2011

13. B. Fan and G. Qi, "Modeling of Product Family Stricture and Module Analysis Method based on Complex Network," Chinese Journal of Mechanical Engineering, Vol. 43, No. 3, pp. 187-186, 2007

14. Y. Li, Z. Wang, L. Zhang, X. Chu, and D. Xue, "Function Module Partition for Complex Products and Systems based on Weighted and Directed Complex Networks," Journal of Mechanical Design, Vol. 139, No. 2, pp. 021101, 2017

15. X. Lian, Y. Yang, and J. Wang, "Research on Complex Product Design Change Propagation based on Complex Networks," in Proceedings of International Conference on Industrial Technology and Management, IEEE, pp. 80-84, 2017

16. W. Zhang, Q. Zhang, and H. Karimi, "Seeking the Important Nodes of Complex Networks in Product R\&D Team based on Fuzzy AHP and TOPSIS," Mathematical Problems in Engineering, No. 1, 2013

17. Q. Li, T. Zhou, L. Lü, and D. Chen, "Identifying Influential Spreaders by Weighted LeaderRank," Physica A Statistical Mechanics \& Its Applications, Vol. 404, No. 24, pp. 47-55, 2013

18. R. Yang, B. H. Wang, J. Ren, W. J. Bai, Z. W. Shi, W. X. Wang, et al., "Epidemic Spreading on Heterogeneous Networks with Identical Infectivity," Physics Letters A, Vol. 364, No. 3-4, pp. 189-193, 2006

19. L. Lv, D. Chen, X. L. Ren, Q. M. Zhang, Y. C. Zhang, and T. Zhou, "Vital Nodes Identification in Complex Networks," Physics Reports, Vol. 650, pp. 1-63, 2016

20. W. Chen, L. V. Lakshmanan, and C. Castillo, "Information and Influence Propagation in Social Networks," Synthesis Lectures on Data Management, Vol. 5, No. 4, pp. 1-177, 2013

21. R. Albert, I. Albert, and G. L. Nakarado, "Structural Vulnerability of the North American Power Grid," Physical Review E Statistical Nonlinear \& Soft Matter Physics, Vol. 69, No. 2, pp. 025103, 2004

22. F. Radicchi, "Who is the Best Player Ever? A Complex Network Analysis of the History of Professional Tennis," Plos One, Vol. 6, pp. e17249, 2011

23. F. Radicchi, S. Fortunato, B. Markines, and A. Vespignani, "Diffusion of Scientific Credits and the Ranking of Scientists," Physical Review E-Statistical, Nonlinear and Soft Matter Physics, Vol. 80, No. 5, pp. 056103, 2009

24. Y. B. Zhou, L. Lv, and M. Li, "Quantifying the Influence of Scientists and their Publications: Distinguishing Between Prestige and Popularity," New Journal of Physics, Vol. 14, No. 3, pp. 33033-33049, 2012

25. T. R. Browning, "Applying the Design Structure Matrix to System Decomposition and Integration Problems: A Review and New Directions," IEEE Transactions on Engineering Management, Vol. 48, No. 3, pp. 292-306, 2002

26. A. Yassine and D. Braha, "Complex Concurrent Engineering and the Design Structure Matrix Method," Concurrent Engineering, Vol. 11, No. 3, pp. 165-176, 2003

27. D. Tang, R. Zhu, J. Tang, R. Xu, and R. He, "Product Design Knowledge Management based on Design Structure Matrix," Advanced Engineering Informatics, Vol. 24, No. 2, pp. 159-166, 2010

28. Y. Fu, M. Li, and F. Chen, "Impact Propagation and Risk Assessment of Requirement Changes for Software Development Projects based on Design Structure Matrix," International Journal of Project Management, Vol. 30, No. 3, pp. 363-373, 2012

29. D. Chen, L. Lü, M. S. Shang, Y. C. Zhang, and T. Zhou, "Identifying Influential Nodes in Complex Networks," Physica A Statistical Mechanics \& Its Applications, Vol. 391, No. 4, pp. 1777-1787, 2012

30. B. S. Brin and L. Page, "The Anatomy of a Large Scale Hypertextual Web Search Engine," Computer Networks and ISDN Systems, Vol. 30, No. 1, pp. 107-117, 1998

31. L. Lv, Y. C. Zhang, H. Y. Chi, and Z. Tao, "Leaders in Social Networks, the Delicious Case," Plos One, Vol. 6, No. 6, pp. e21202, 2011

32. M. Pinzger, N. Nagappan, and B. Murphy, "Can Developer-Module Networks Predict Failures," in Proceedings of ACM SIGSOFT Symposium on the Foundations of Software Engineering, SIGSOFT'08, pp. 2-12, ACM Press, 2008

33. J. Xuan, H. Jiang, Z. Ren, and W. Zou, "Developer Prioritization in Bug Repositories," in Proceedings of 201234 th International Conference on Software Engineering, ICSE'12, pp. 25-35, IEEE Press, 2012

34. Y. Li, Z. Wang, X. Zhong, and F. Zou, "Identification of Influential Function Modules within Complex Products and Systems based on Weighted and Directed Complex Networks," Journal of Intelligent Manufacturing, pp. 1-16, 2018 